\title{
Expression of Lilium pumilum SHORT VEGETATIVE PHASE gene in transgenic Nicotiana tabacum delays flower bud differentiation
}

\author{
Liuhui Yang*, Wang Wang*, Xiaoxia Su, Yiru Yang, Yunwei Zhou and Miao He \\ College of Landscape Architecture, Northeast Forestry University, Harbin, China \\ * These authors have contributed equally to this work.
}

\begin{abstract}
Summary
This study is focused on the function of the SHORT VEGETATIVE PHASE gene. Based on morphological observation of flower buds, flower bud differentiation occurs during low-temperature storage of Lilium pumilum bulbs and progresses as the duration of low-temperature storage time. Real-time quantitative PCR analysis showed that the relative expression level of the LpSVP gene first increases and then decreases during low temperature storage. Morphological observation combined with gene expression analyses suggested that $L p S V P$ acts as a repressor of flower meristem identification. To further characterise the function of the LPSVP gene, we constructed the plant expression vector pBI121-LpSVP-GFP, and the fusion protein was transferred into wild-type tobacco via Agrobacterium-mediated transformation of leaf discs. The resulting transgenic plants exhibited delayed flowering and a more lax inflorescence compared with the wild-type plants. This study lays the foundation for further study of the function of the LPSVP gene.
\end{abstract}

Keywords

delayed flowering, genetic transformation, lowtemperature storage, real-time quantitative PCR, temperature, SVP gene

\section{Introduction}

The SHORT VEGETATIVE PHASE gene (SVP) encodes an important $M A D S$-box transcription factor that negatively regulates flowering. It contains eight introns and nine exons, an open reading frame of $723 \mathrm{bp}$, which encodes 240 amino acids, and has a molecular weight of $28.9 \mathrm{kDa}$ (Shinan and Xinchun, 2014). SVP is downstream of FVE and FCA in an autonomous pathway, and its expression is affected by environmental factors such as temperature and light. The main regulatory function of $S V P$ is to maintain plant vegetative growth and to inhibit the flowering process without altering the effects of vernalization and photoperiod on flowering time (Li et al., 2008). Among the MADS-box transcription factors, $S V P$ is most closely related, in an evolutionary sense, to AGL24, both belonging to the STMADS11 subfamily (Yu et al., 2002). However, these two transcription factor genes operate in different directions in flower formation. SVP maintains the growth of vegetative buds, whereas AGL24 promotes the identity of inflorescence tissue in Arabidopsis. The svp mu-

\section{Significance of this study}

What is already known on this subject?

- The SVP gene inhibits flowering, by protein-protein interactions.

What are the new findings?

- Flower bud differentiation occurred in bulbs of Lilium pumilum during low-temperature storage time. The expression level of the SVP gene first increased and then decreased during the entire storage process. Transformation of tobacco with the LpSVP gene resulted in delayed flowering.

What is the expected impact on horticulture?

- Manipulation of LpSVP could help to develop improved Lilium cultivars.

tant blooms earlier than does the wild-type, whereas constitutive expression of SVP delays flowering in Arabidopsis, with the development of floral phenotypes being influenced by the transformation of flower buds into vegetative buds (Gregis et al., 2006). Recent studies have shown that the inhibitory effect of SVP on flowering is mediated through an interaction with $F L C$, an effective flowering suppressor gene (Li et al., 2008). The svp and flc mutants bloom early and have increased FT and SOC1 mRNA levels (Hartmann et al., 2000). SVP may express a regulatory mechanism for flower meristem recognition conserved across both monocotyledonous and dicotyledonous plants that involves an interaction between SVP and AP1 MADS-box proteins. Arabidopsis SVP and AGL24 form heterodimers with the MADS-domain protein AP1 and inhibit the B and C floral organ characteristic genes by recruiting the SEUSS-LEUNIG co-repressor complex (Posé et al., 2012). Subsequently, the expression of SVP is repressed by SEP3 and then works with LFY to activate B and C flower organ genes (Kaufmann et al., 2009). The interaction between SVP and AP1 proteins is also important for floral development in cereals and other grasses. Flowering time was accelerated in transgenic tobacco plants exhibiting ectopic expression of soybean GmSVP1 and the development of stamens, petals, and flower buds were abnormal (Zhang et al., 2016). As described above, SVP has a variety of regulatory effects on flower development.

In recent years, the functions of the SVP gene in flower development have been widely studied. Many SVP genes have been identified and cloned from ornamental plants, and the flowering mechanism of many plants has been revealed. However, it is not clear whether SVP plays a role in lily flow- 
er development. Lilium pumilum has flowers with bright colours and is of great ornamental and horticultural value. Studying the role of the SVP gene in lily flower bud differentiation could help to further improve this ornamental plant. In current studies, we found that the LpSVP sequence has the highest homology with the SVP protein of Lilium formosanum $\times$ Lilium longiflorum, and the homology reaches 99\%; RT-qPCR analysis of $L p S V P$ gene expression over five stages (flower bud stage I, flower bud stage II, flower bud stage III, flower bud end stage, full bloom stage) of flower development indicated that the expression level of the LpSVP gene first increased and then decreased during the flower development process; The expression level of phase II reached the maximum, and the full bloom stage reached the minimum (Wang et al., 2018).

In this study, L. pumilum was used as the test material, and the biological function of SVP was analyzed by transforming tobacco with a constructed plant expression vector. This study lays the foundation for further exploration of the molecular mechanisms of SVP in flower development and provides a theoretical basis for improving the control of flowering in an important ornamental plant, using genetic engineering.

\section{Materials and methods}

\section{Materials}

Intact and damage-free L. pumilum bulbs with a circumference of 4-6 cm were selected from the nursery at the Northeast Forestry University, Harbin, China. The bulbs were washed with purified water, soaked in $50 \%$ carbendazim wettable powder diluted 1,000 times for 30 minutes, rinsed with water and air dried. Finally, they were mixed with disinfected vermiculite (to maintain a relatively consistent humidity), placed in plastic pots, sealed with plastic wrap (perforated to allow gas exchange) and storage $4^{\circ} \mathrm{C}$. Samples were taken after 0, 30, 60 and $90 \mathrm{~d}$ low-temperature and used as test materials, labelled S1, S2, S3 and S4, respectively. Paraffin sections were prepared, and RNA was extracted to construct a sequencing library.

\section{Methods}

1. Morphological observation off flower bud differentiation during cold storage. Bulbs were sampled at each of the four time periods. The stem tips and scales were fixed with Formalin-acetic acid-alcohol fixative and sectioned by routine paraffin sectioning. After staining with dyeing agent (Safranin and Fast Green), stem tips and scales were observed and photographed under a Leica optical microscope (DM500, Germany). At the same time, flower bud differentiation was observed using an entity microscope.

2. Analysis of the relative expression levels of $L p S V P$ at different storage periods. Total RNA was extracted from bulb samples which had been stored under low-temperature conditions for each of the four time periods. The LpSVP gene (GenBank accession number MF693882) specific primers were designed using Primer-BLAST software on the NCBI website (primer pair F: 5'-AGACCAGTGGCATAGCTTGT-3' and R: 5'-GGGAAGCAGGTGGTAACTGT-3'). The internal reference gene, Lilium-Actin (Yun et al., 2013) (GenBank accession number JX826390), had been used in a previous study (Yun et al., 2013) and amplified with internal reference primers (F: 5'-TGCTGACCGTATGAGCAAGG-3' and R: 5'-GACGATGGCTGGACCAGATT-3'). Reverse transcription was performed, and the reverse transcribed cDNA was diluted five-fold and used as the template for RT-qPCR. RT-qPCR was performed on a Roche LightCycler 96 using the SYBR Green Master Mix (Toyobo, Japan). Three-step fluorescence signal collection was performed (Predenaturation at $95^{\circ} \mathrm{C}$ for $30 \mathrm{~s}$; three steps at $95^{\circ} \mathrm{C}$ for $5 \mathrm{~s}, 60^{\circ} \mathrm{C}$ for $15 \mathrm{~s}$ and $72^{\circ} \mathrm{C}$ for $30 \mathrm{~s}$ for 40 cycles; dissolution at $95^{\circ} \mathrm{C}$ for $10 \mathrm{~s}, 65^{\circ} \mathrm{C}$ for $60 \mathrm{~s}, 97^{\circ} \mathrm{C}$ for $1 \mathrm{~s}$; Cooling at $37^{\circ} \mathrm{C}$ for $30 \mathrm{~s}$ ), and expression levels were determined using the $2^{-\Delta \Delta \mathrm{Ct}}$ method (Livak and Schmittgen, 2001).

3. Construction of the pBI121-LpSVP-GFP plant expression vector. The plasmid was diluted 100 -fold and used as a template after the correct $L p S V P$ sequence was confirmed by DNA sequencing. Specific primers to introduce KpnI (NEB, MA, USA) and SpeI (NEB, MA, USA) digestion sites (F: 5'-GGTACCATGGCGAGGGAGAAGATA-3' and R: 5'-ACTAGTTACATATGCATACCCTAA-3') were used for PCR amplification. Plasmids with restriction enzyme sites and the pBI121-MCSGFP vector with a Green Fluorescent Protein (GFP) reporter gene were digested with $\mathrm{KpnI}+\mathrm{SpeI}$ in water at $37^{\circ} \mathrm{C}$ for 30 min, purified by agarose gel electrophoresis and recovered, and T4-ligated overnight to construct the pBI121-LpSVP-GFP plant expression vector.

4. Infected tobacco leaf disc transformation. The plant expression vector pBI121-LpSVP-GFP was transformed into Agrobacterium using electric shock. Agrobacterium recombinant plasmids were identified by PCR, and tobacco tissue was transformed by the leaf disc method (Endo et al., 2001), using plant tissue culture to obtain transgenic positive plants. 5. Identification of transgenic plants at the molecular level. Total DNA was extracted from wild-type and transgenic tobacco using the DNAquick Plant System kit (Tiangen, China). Wild-type tobacco total DNA and water were used as negative controls. The pBI121-LpSVP-GFP plasmid was used as the positive control. PCR amplification was performed with the target gene-specific primers. The amplification reaction was performed using Gotaq DNA polymerase (Promega, USA). Amplification procedures were carried out as follows: predenaturation at $95^{\circ} \mathrm{C}$ for $2 \mathrm{~min}$; denaturation at $95^{\circ} \mathrm{C}$ for $30 \mathrm{~s}$, annealing $65-0.5^{\circ} \mathrm{C} /$ cycle for $30 \mathrm{~s}$, extension at $72^{\circ} \mathrm{C}$ for $1 \mathrm{~min}, 40$ cycles; final extension at $72^{\circ} \mathrm{C}$ for $5 \mathrm{~min}$; and then held at $4^{\circ} \mathrm{C}$. The amplified product was detected by electrophoresis on agarose gel.

6. Phenotypic observation of transgenic tobacco. Wildtype and transgenic tobacco plants with consistent growth were selected and transplanted into soil. The first flower opening time was monitored and flower pattern differences were observed and photographed. The maximum diameter of the corolla tube, the mean length of the pistil and stamen, the maximum diameter of the ovary, the maximum length of the handle, the length of the corolla, the plant height, the number of leaves and the number of flowers were all measured. Excel 2016 and SPSS 22.0 (IBM, USA) were used for data and statistical analysis.

\section{Results}

\section{Observations of flower bud differentiation}

Scanning electron and light microscopy observations revealed that the degree of flower bud differentiation of L. pumilum increased gradually with prolonged storage time. In bulbs stored at low-temperature for $0-30 \mathrm{~d}$, the growth cone had a certain height and width. The young leaves on both sides and small spherical bulges could be seen on the inflorescence axis, features which indicate small floral primordium (Figure 1a and 1d). After $60 \mathrm{~d}$ of storage, the growth cones had swelled, but the difference was not very signifi- 


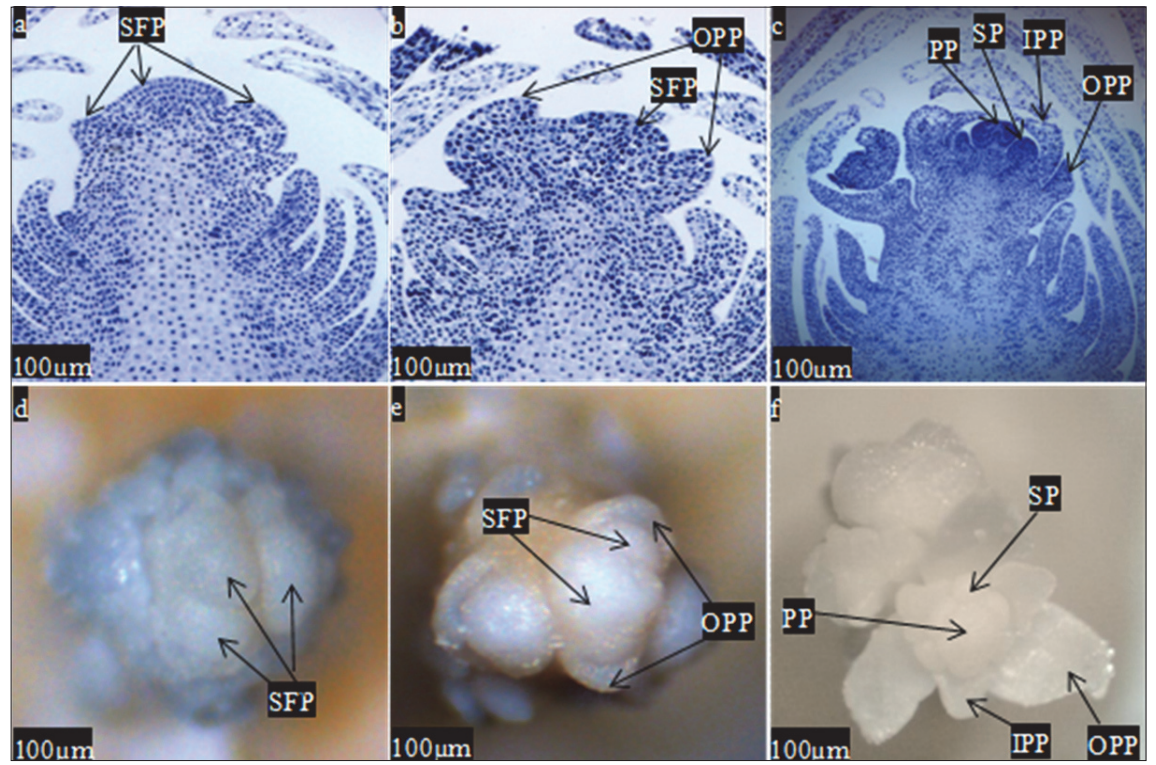

FIGURE 1. Observation of flower bud differentiation in Lilium pumium. SFP is the small floral primordium; OPP is the outer perianth primordium; IPP is the inner perianth primordium; SP is the stamen primordium; and PP is the pistil primordium. cant compared to day 0 . The leaves had further elongated. At $60 \mathrm{~d}$, the small floral primordium of the floret was further developed. The lower edge and the center of the growth cone had raised into a crescent shape, forming the outer perianth primordium (Figure 1b and 1e). After $90 \mathrm{~d}$ of storage, the growth cones were markedly enlarged as compared with $0 \mathrm{~d}$. Small protrusions appeared on the inner side of the outer perianth to form the inner perianth primordium at $90 \mathrm{~d}$. Small protrusions corresponding to the stamen primordia, were also observed on the inner side of the perianth. The central growth point on the inner side of the primordia had a relatively large protrusion, which was the pistil primordium (Figure 1c and 1f). At this period, flower bud differentiation had reached maturity. By the end of the period of low-temperature storage, the structure of the flower bud completely differentiated.

\section{Quantitative LpSVP expression analysis at different periods of low-temperature storage}

RT-qPCR was used to analyze the relative expression of the LPSVP gene in the bulbs following storage at low-temperature at the four periods (Figure 2). The expression of LpSVP first increased and then decreased. Maximum relative expression was observed at $\mathrm{S} 2$, which was 1.43 times that at $\mathrm{S} 1$. The relative expression decreased continuously from S2 to S4, with the S4 relative expression being 0.27 times that

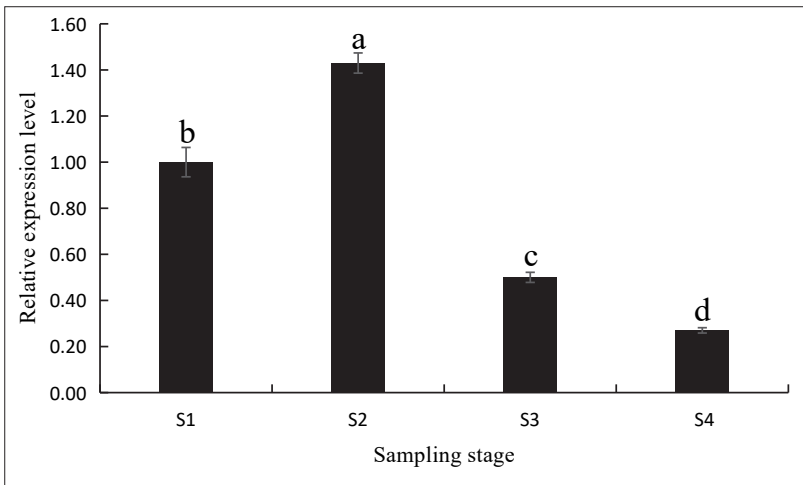

FIGURE 2. The relative expression of the LpSVP gene in Lilium pumilum bulbs stored for different periods of time at low temperature. Different lowercase letters indicate significant differences at the $\mathrm{p}<0.05$ level. of S1 expression. All relative gene expression differences between the sampling were significant.

\section{Plant expression vector construction}

The double enzyme digestion yielded the correct band size. The product was recovered from the agarose gel and ligated using $\mathrm{T} 4$ ligase at $16^{\circ} \mathrm{C}$ overnight to construct the recombinant vector. The vector containing the gene of interest was subjected to double enzyme digestion to verify the insert. Two bands appeared after double digestion and the sizes of the target band (675 bp) was correct. The plasmid was sent to Bosch Biosciences Company for sequencing. The sequencing results were consistent with the target gene, indicating that the plant expression vector had been successfully constructed.

\section{Observation of the differentiation of transgenic tobacco explants}

Non-transgenic tobacco and transgenic tobacco explants were cultured under the same conditions. The non-transgenic tobacco leaves gradually died on the selective medium, but the transgenic tobacco survived. For a period of time, callus grew on the edges of the transgenic leaves, and then adventitious buds formed. After subculture, adventitious buds were cut and transferred to rooting medium to induce rooting (Figure 3).

\section{Molecular confirmation of transgenic tobacco}

Electrophoretic analysis of the amplified products revealed that no target bands appeared in the negative control, but a single band appeared as the amplified product in transgenic tobacco. The size of the band was the same size as the target gene. These results showed that the LpSVP gene had been successfully transferred into tobacco (Figure 4) and five transgenic plants were obtained.

\section{Phenotypic observation of transgenic tobacco plants}

The flowering date, flower and inflorescence of transgenic and wild-type tobacco were analyzed and compared (Figure 5). Summary statistics and results of statistical analyzes are shown in Table 1. Flowering of tobacco transformed with LpSVP occurred approximately $22 \mathrm{~d}$ later than that of wildtype tobacco, indicating that ectopic expression of the LpSVP gene delayed the flowering time of tobacco. The branching 
of the transgenic tobacco inflorescence also increased, and the inflorescence was slightly more lax overall, whereas the wild-type inflorescence was relatively compact.

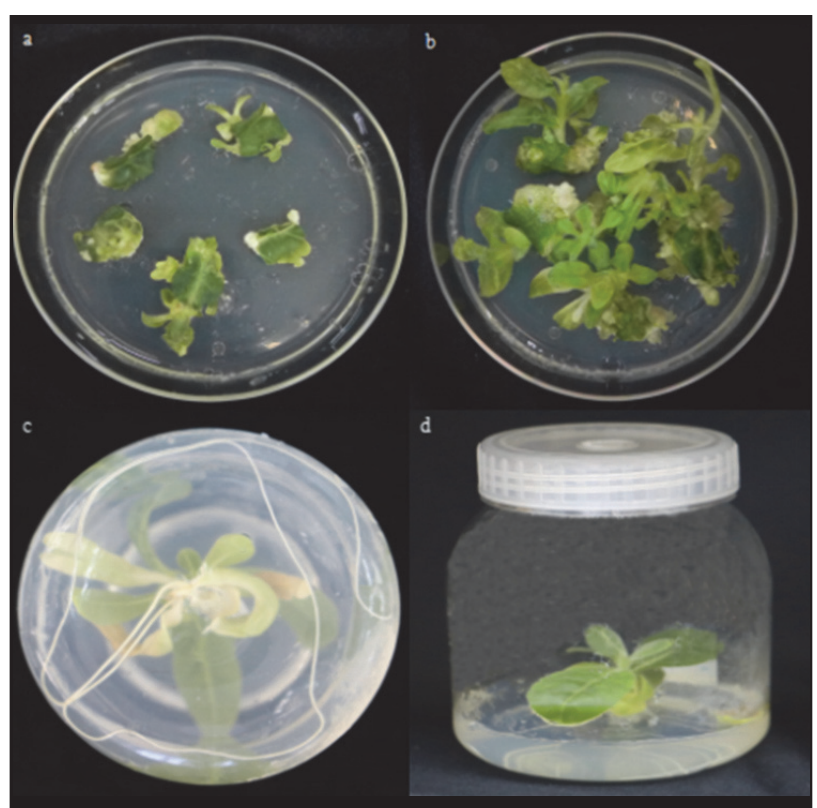

FIGURE 3. Observations on differentiation from transgenic tobacco explants. a) Transgenic tobacco leaves developed callus; b) Transgenic tobacco leaves developed adventitious buds; c) Adventitious buds of transgenic tobacco were induced to form roots; d) Adventitious buds of transgenic tobacco grew into plants.

\section{Discussion}

Leaf bud physiology and tissue state transforms into flower bud physiology and tissue state when plants reach a certain growth stage. This biological process is called flower bud differentiation (Wu et al., 2007). The duration of dormancy and the process of flower bud development also differs greatly in different lily species (Fukai and Goi, 2001; Tu et al., 2005). Jimin et al. (1985) classified the process of flower bud differentiation of L. longiflorum into five periods, which included no visible differentiation, visible floral primordial, visible outside petal, visible inside petal, and visible and stamen and pistil. Other authors (Ning et al., 2008) have divided the flower bud differentiation process of Lilium formolongi 'Raizan' into on differentiation, initial differentiation, inflorescence primordium, small floral primordium differentiation, floral organ differentiation, and inflorescence formation phases. Guo et al. (2006) performed morphological analysis of three lily varieties, namely 'Snow Queen' (Longiflorum hybrid), 'Siberia' (Oriental hybrid) and 'Gondelina' (Asiatic hybrid). They categorized the differentiation process into five periods, namely, no visible differentiation, visible floral primordial, visible petal, visible stamen and pistil, visible the whole inflorescence. Liu et al. (2015) classified the flower bud differentiation of L. pumilum into floret primordium differentiation, outer perianth primordium differentiation, inner perianth primordium differentiation, and stamen and pistil primordium differentiation phases.

In our study, we found that the degree of flower bud differentiation progressed with the duration of storage time at low temperatures. After low-temperature storage for $30 \mathrm{~d}$, the floret primordium differentiated on the inflorescence

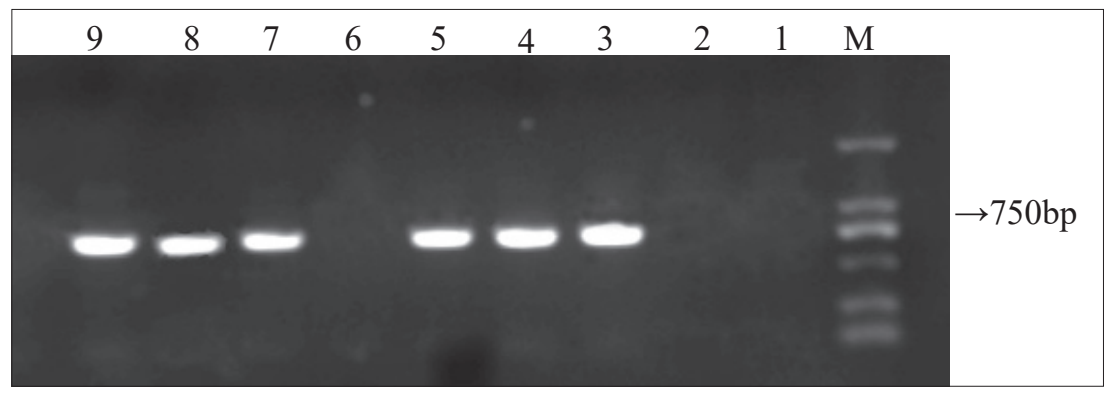

FIGURE 4. Electrophoresis of PCR products on $1 \%$ agarose gel, identifying transgenic tobacco plants. 1 is a negative control with water as a template; 2 is a negative control with wild-type tobacco as the template; 3 is a positive control with pBI121-LpSVP-GFP plasmid; 4-9 are the PCR results of transgenic pBI121-LpSVP-GFP tobacco.

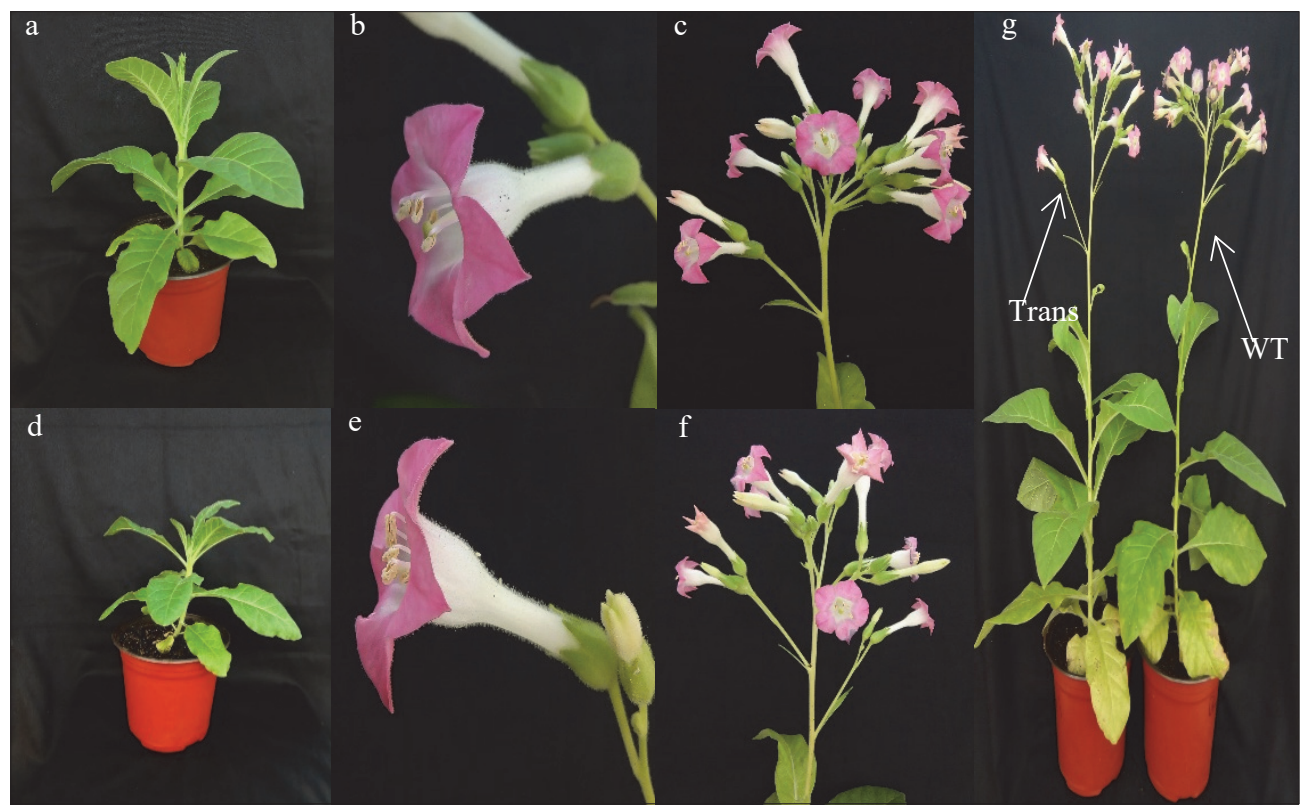

FIGURE 5. Phenotypic observations of transgenic tobacco and wildtype tobacco. a), b), c), WT) are wild-type tobacco; d), e), f), Trans) are transgenic tobacco. 
TABLE 1. Phenotypic characters of transgenic LpSVP-expressing and wild-type tobacco.

\begin{tabular}{lcc}
\hline Phenotype & Wild-type tobacco & Transgenic tobacco \\
\hline Time from planting to the first flower opening date (days) & $37.0 \pm 1.84 \mathrm{~b}$ & $59.8 \pm 2.71 \mathrm{a}$ \\
Number of flowers in the inflorescence $(n)$ & $38.60 \pm 2.11 \mathrm{a}$ & $36.60 \pm 1.91 \mathrm{a}$ \\
Leaf number $(n)$ & $15.80 \pm 1.24 \mathrm{a}$ & $15.4 \pm 1.29 \mathrm{a}$ \\
Plant height $(\mathrm{cm})$ & $50.14 \pm 2.07 \mathrm{a}$ & $49.44 \pm 1.81 \mathrm{a}$ \\
Maximum diameter of corolla tube $(\mathrm{cm})$ & $1.0 \pm 0.03 \mathrm{a}$ & $1.0 \pm 0.03 \mathrm{a}$ \\
Stamen length $(\mathrm{cm})$ & $4.12 \pm 0.04 \mathrm{a}$ & $4.10 \pm 0.03 \mathrm{a}$ \\
Pistil length $(\mathrm{cm})$ & $4.10 \pm 0.03 \mathrm{a}$ & $4.08 \pm 0.04 \mathrm{a}$ \\
Maximum diameter of ovary $(\mathrm{cm})$ & $0.40 \pm 0.0 \mathrm{a}$ & $0.40 \pm 0.0 \mathrm{a}$ \\
The maximum length of the handle $(\mathrm{cm})$ & $1.88 \pm 0.04 \mathrm{a}$ & $1.86 \pm 0.05 \mathrm{a}$ \\
Inflorescence branch habit & Compact & Lax \\
Corolla length $(\mathrm{cm})$ & $6.08 \pm 0.04 \mathrm{a}$ & $6.08 \pm 0.04 \mathrm{a}$ \\
\hline
\end{tabular}

Note: The data in the table are mean average \pm standard deviation; $d$ : days.

Different lowercase letters indicate significant differences between the samples in the same row at the $p<0.05$ level.

axis. After low-temperature storage for $60 \mathrm{~d}$, the outer perianth primordium differentiated, and after low-temperature storage for $90 \mathrm{~d}$, the differentiation of inner perianth primordium, stamen and pistil primordium were complete. Our findings were consistent with those of Liu et al. (2015). Li et al. (2017) found that the expression levels of PmSVP1 and PmSVP2 decreased during the process of Prunus mume flower bud differentiation, which indicated that these genes played important roles in flower bud differentiation. The downregulation of the LPSVP gene corresponded to the flower bud differentiation process, and the decrease in the SVP transcript level is accompanied by rapid accumulation of $B$ and $\mathrm{C}$ gene transcripts. These regulatory mechanisms maintain flower meristem identification during dormancy (Gregis et al., 2009). The morphological changes during flower bud differentiation are closely related to changes in gene expression. We concluded that LPSVP acts as a repressor of flower meristem recognition, and that downregulation of $L p S V P$ might activate the flower development-associated genes, therefore promoting flower bud differentiation (Figure 6).

At least 80 gene loci have been identified to be involved in the flowering process of plants based on studies of the model plant Arabidopsis (Levy and Dean, 1998), of which more than 20 loci play regulatory roles in by delaying flowering. SVP is one of the key repressor genes, which determines the duration of the plant vegetative period (Levy and Dean, 1998). The svp mutant blooms earlier, but constitutive expression of SVP in plants delays flowering (Hartmann et al., 2000). The regulatory mechanisms involve the interaction of protein complexes at various plant development stages. SVP and $F L C$ form a flowering repressor complex during vegetative growth, down-regulating the expression of SOC1 and FT, and thereby inhibiting flowering (Lee et al., 2007b; Li et al., 2008). Previous studies have reported that four MADS-box family genes, namely SOC1, SVP, AGL24 and SEP4, form a conserved genetic pathway which determines the inflorescence structure of both Arabidopsis and rice (Liu et al., 2013). We found that the flowering time in transgenic LpSVP tobacco occurred later than in the wild-type, a finding which is consistent with those of previous studies (Jaudal et al., 2014; Lee et al., 2007a). We also found that there were more inflorescence branches in the transgenic tobacco, so the overall structure of the inflorescence was slightly altered compared with to the wild-type.

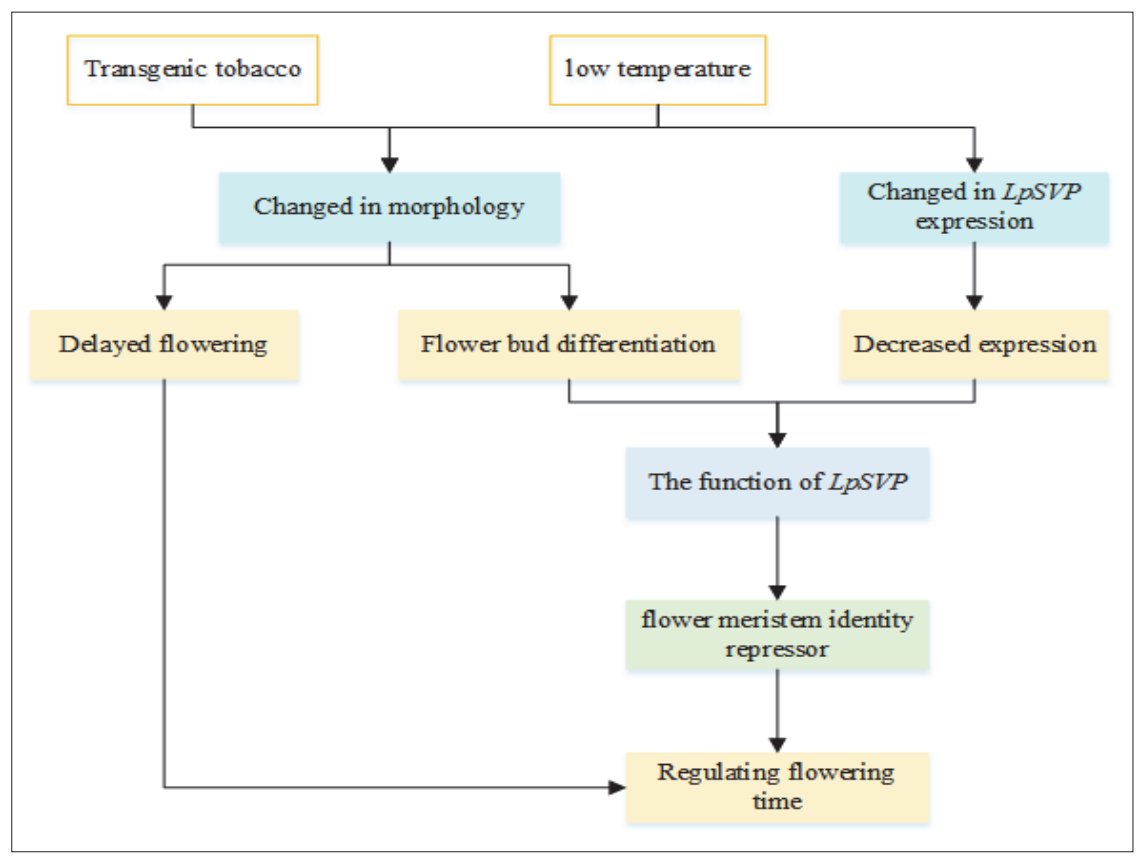

FIGURE 6. The regulatory mechanism of the LPSVP gene in flower development. 


\section{Conclusion}

We found that L. pumilum flower bud differentiation was accompanied by down-regulated expression of $L p S V P$, which suggests that LPSVP may act as a repressor of flower meristem identification. Through phenotypic characterization of transgenic tobacco, we further verified the important regulatory role of $L p S V P$ in plant flower development. The functional identification and analysis of LpSVP lays the foundation for greater understanding of the molecular mechanisms of flower development.

\section{Acknowledgments}

This study was financially supported by the National Natural Science Foundation of China (No. 31470698).

\section{References}

Endo, S., Kasahara, T., Sugita, K., Matsunaga, E., and Ebinuma, H. (2001). The isopentenyl transferase gene is effective as a selectable marker gene for plant transformation in tobacco (Nicotiana tabacum cv. Petite Havana SRI). Plant Cell Reports 20, 60-66. https://doi. org/10.1007/s002990000279.

Fukai, S., and Goi, M. (2001). Floral initiation and development in Lilium longiflorum Thunb. Techn. Bull. Fac. Agric. 53, 31-34.

Gregis, V., Sessa, A., Colombo, L., and Kater, M.M. (2006). AGL24, SHORT VEGETATIVE PHASE, and APETALA1 redundantly control AGAMOUS during early stages of flower development in Arabidopsis. Plant Cell 18, 1373-1382. https://doi.org/10.1105/tpc.106.041798.

Gregis, V., Sessa, A., Dorca Fornell, C., and Kater, M.M. (2009). The Arabidopsis floral meristem identity genes AP1, AGL24 and SVP directly repress class B and C floral homeotic genes. Plant J. 60, 626637. https://doi.org/10.1111/j.1365-313X.2009.03985.x.

Guo, R., Zhao, X., Wang, W., and Chen, W. (2006). Observation of morphological changes of Lily bulb in bud differentiation periods. J. Shenyang Agric. Univ. 37, 31-34.

Hartmann, U., Hohmann, S., Nettesheim, K., Wisman, E., Saedler, H., and Huijser, P. (2000). Molecular cloning of SVP: A negative regulator of the floral transition in Arabidopsis. Plant J. 21, 351-360. https:// doi.org/10.1046/j.1365-313x.2000.00682.x.

Jaudal, M., Monash, J., Zhang, L., Wen, J., Mysore, K.S., Macknight, R., and Putterill, J. (2014). Overexpression of Medicago SVP genes causes floral defects and delayed flowering in Arabidopsis but only affects floral development in Medicago. J. Exp. Bot. 65, 429-442. https://doi.org/10.1093/jxb/ert384

Jimin, H., Jianying, Y., and Guodong, L. (1985). Observation on the flower bud differentiation process of Lilium longiflorum. Acta Hortic. Sinica 12, 203-206.

Kaufmann, K., Muiño, J.M., Jauregui, R., Airoldi, C.A., Smaczniak, C., Krajewski, P., and Angenent, G.C. (2009). Target genes of the MADS transcription factor SEPALLATA3: Integration of developmental and hormonal pathways in the Arabidopsis flower. PLOS Biology 7, e1000090. https://doi.org/10.1371/journal.pbio.1000090.

Lee, J.H., Park, S.H., Lee, J.S., and Ahn, J.H. (2007a). A conserved role of SHORT VEGETATIVE PHASE (SVP) in controlling flowering time of Brassica plants. Biochim. Biophys. Acta - Gene Structure and Expression 1769, 455-461. https://doi.org/10.1016/j. bbaexp.2007.05.001.

Lee, J.H., Yoo, S.J., Park, S.H., Hwang, I., Lee, J.S., and Ahn, J.H. (2007b). Role of $S V P$ in the control of flowering time by ambient temperature in Arabidopsis. Genes Dev. 21, 397-402. https://doi.org/10.1101/ gad.1518407.

Levy, Y.Y., and Dean, C. (1998). The transition to flowering. Plant Cell 10, 1973-1989. https://doi.org/10.1105/tpc.10.12.1973.
Li, D., Liu, C., Shen, L., Wu, Y., Chen, H., Robertson, M., Helliwell, C.A., Ito, T., Meyerowitz, E., and Yu, H. (2008). A repressor complex governs the integration of flowering signals in Arabidopsis. Developm. Cell 15, 110-120. https://doi.org/10.1016/j.devcel.2008.05.002.

Li, Y., Zhou, Y., Yang, W., Cheng, T., Wang, J., and Zhang, Q. (2017). Isolation and functional characterization of SVP-like genes in Prunus mume. Sci. Hortic. 215, 91-101. https://doi.org/10.1016/j. scienta.2016.12.013.

Liu, C., Teo, Z.W.N., Bi, Y., Song, S., Xi, W., Yang, X., Yin, Z., and Yu, H. (2013). A conserved genetic pathway determines inflorescence architecture in Arabidopsis and rice. Developm. Cell 24, 612-622. https://doi.org/10.1016/j.devcel.2013.02.013.

Liu, F., Tian, Z., Cai, Y., Zhang, Y., and Zhou, Y. (2015). Change in starch grains and flower bud differentiation of Lilium pumilum bulbs during breaking of dormancy under refrigerated conditions. Acta Pratacult. Sinica $24,154-162$

Livak, K.J., and Schmittgen, T.D. (2001). Analysis of relative gene expression data using real-time quantitative PCR and the $2^{-\Delta \Delta C \mathrm{~T}}$ method. Methods 25, 402-408. https://doi.org/10.1006/ meth.2001.1262.

Ning, Y., Ling, M., Tao, J., Yang, M., and Wei, P. (2008). Morphological observation on flower bud differentiation of Lilium $\times$ formolongi bulb. Acta Hortic. Sinica 35, 1368-1372.

Posé, D., Yant, L., and Schmid, M. (2012). The end of innocence: flowering networks explode in complexity. Curr. Opin. Plant Biol. 15, 45-50. https://doi.org/10.1016/j.pbi.2011.09.002.

Shinan, L., and Xinchun, L. (2014). Advance of studies on SVP gene in plants. Biotechnol. Bull., 9-13.

Tu, S., Mu, D., and Liu, C. (2005). The physiological and biochemical changes during flower bud differentiation of different lily cultivars. Chinese Agric. Sci. Bull. 21, 207-209.

Wang, W., Xiaoxia, S., Liuhui, Y., and Yunwei, Z. (2018). Cloning and expression analysis of LpSVP gene from Lilium pumilum. J. Northwest Forestry Univ. 33, 89-94.

Wu, L., Tang, Q., Yin, H., and Chen, L. (2007). Flower bud differentiation mechanism of ornamental plants. Letters Biotechnol. 18, 1064-1067.

Yu, H., Xu, Y., Tan, E.L., and Kumar, P.P. (2002). AGAMOUS-LIKE 24, A dosage-dependent mediator of the flowering signals. Proc. Natl. Acad. Sci. USA 99, 16336-16341. https://doi.org/10.1073/ pnas.212624599.

Yun, L., Su-xia, Y., Hui-ying, F., Lei-feng, X., Ying-ying, Y., Chun, L., and Jun, M. (2013). Cloning and expression analysis of Actin gene (lilyActin) from Lily. Acta Hortic. Sinica 40, 1318-1326.

Zhang, H., Yan, H., Zhang, D., and Yu, D. (2016). Ectopic expression of a soybean $S V P$-like gene in tobacco causes abnormal floral organs and shortens the vegetative phase. Plant Growth Regul. 80, 345-353. https://doi.org/10.1007/s10725-016-0173-z.

Received: Jan. 3, 2019

Accepted: Oct. 18, 2019

\section{Address of authors:}

Liuhui Yang, Wang Wang, Xiaoxia Su, Yiru Yang, Yunwei Zhou* and Miao $\mathrm{He}^{* *}$

College of Landscape Architecture, Northeast Forestry University, Harbin 150040, China

* Corresponding author; E-mail: dlzhyw@nefu.edu.cn

** Corresponding author; E-mail: hemiao@nefu.edu.cn 